\title{
The LAGO Space Weather Program: Directional Geomagnetic Effects, Background Fluence Calculations and Multi-Spectral Data Analysis
}

\author{
H. Asorey ${ }^{1,2,3}$, S. Dasso ${ }^{4,5}$, L.A. Núñez ${ }^{3,6}$, Y. Peréz ${ }^{6}$, C. Sarmiento-Cano*3, M. \\ Suárez-Durán ${ }^{33}$, for The LAGO Collaboration ${ }^{7}$ \\ ${ }^{1}$ Laboratorio Detección de Partículas y Radiación, Instituto Balseiro y Centro Atómico \\ Bariloche, S.C. de Bariloche, Argentina. \\ ${ }^{2}$ Sede Andina, Universidad Nacional de Río Negro, S.C. de Bariloche, Argentina. \\ ${ }^{3}$ Escuela de Física, Universidad Industrial de Santander, Bucaramanga, Colombia. \\ ${ }^{4}$ Instituto de Astronomía y Física del Espacio (UBA-CONICET), Buenos Aires, Argentina. \\ ${ }^{5}$ Dept. de Ciencias de la Atmósfera y los Océanos and Dept. de Física, Universidad de Buenos \\ Aires, Buenos Aires, Argentina. \\ ${ }^{6}$ Centro de Física Fundamental, Dept. de Física, Universidad de Los Andes, Mérida Venezuela \\ ${ }^{7}$ lagoproject.org, see the full list of members and institutions at lagoproject.org/collab.html \\ e-mail: lago-pielagoproject.org
}

\begin{abstract}
The Latin American Giant Observatory (LAGO) is an extended cosmic ray observatory operating in nine Latin American countries. Within the LAGO framework, several scientific and academic programs are being developed and conducted. One of them, the LAGO Space Weather program, aims at producing real time, high resolution and high quality data of the flux of secondary particles at each site of the LAGO detection network, complementing and expanding present measurements of the influence of solar activity from ground level observations. With the LAGO detection network, transient events or long term modulation effects can be measured simultaneously at places having different geomagnetic rigidity cut-offs and atmospheric reaction levels. As the data acquisition system of LAGO registers the individual energy of all secondaries detected, these transients can furthermore be studied in different energy bands. This program is intensively supported by a complex chain of simulations that accounts for geomagnetic and atmospheric effects and the different detectors response. In this work, the complete simulation chain is described, and the first multi-spectral analysis of the Forbush decrease of March of 2012 is presented.
\end{abstract}

The 34th International Cosmic Ray Conference,

30 July- 6 August, 2015

The Hague, The Netherlands

\footnotetext{
${ }^{*}$ Presenter

† Speaker.
} 


\section{Introduction}

Transient solar ejecta and changes in the global structure of the heliospheric and terrestrial magnetic fields modulate the flux of low energy cosmic rays of galactic origin (GCR). Measure of the temporal variations of this flux provide information about the transport of particles in the inner and outer heliosphere.

Long-term modulations in the flux of GCR are associated with the solar cycle, while short-term are produced by interplanetary transient perturbations in the calm solar wind causing phenomena like Forbush Decreases [1]. Observed from ground level, FDs exhibit asymmetrical structures: fast decreases (several hours) of the cosmic ray flux are followed by smooth recoveries (several days). In some particular cases, they can also present more complex structures originated by intricate interplanetary Coronal Mass Ejections (iCME) phenomena such as its interactions with fast streams of plasma or with other iCMEs.

Simultaneous measurements of the GCR flux modulation at different locations on Earth using the same type of detectors, can provide important information about the global structure of the magnetic cloud reaching the near-Earth space environment. Astroparticles studies in the context of GRBs, Space Weather and background radiation at ground level are the main scientific objectives of the Latin American Giant Observatory (LAGO, previously known as Large Aperture GRB Observatory)[2].

The LAGO Project is being built as an extended Astroparticle Observatory at a regional scale, operated by a collaborative and non-centralized network of institutions from nine Latin American countries (Argentina, Bolivia, Brazil, Colombia, Ecuador, Guatemala, Mexico, Peru and Venezuela) and recent incorporation of institutions from Spain.

The LAGO detection network currently consists of single or small arrays of water Cherenkov detectors $(\mathrm{WCD})$ located at different altitude sites and covering a large range of geomagnetic rigidity cut-offs $\left(R_{c}\right)$ and atmospherics reaction levels [3]. Current status of the LAGO project and information about the sites characteristics are shown in figure 1, covering from Mexico [4] to Antarctic [5].

This paper is organized as follows: in the coming section we describe the LAGO Space Weather (LAGO-SW) program; then geomagnetic and atmospheric simulations are detailed in section 3, showing a procedure to evaluate how the disturbances in the geomagnetic field (GF) can be linked with variations in the flux of secondary particles at ground level. Then the first steps of the multi-spectral analysis are presented by the analysis of a FD observed on March 8th 2012 in a single WCD located in Bariloche, Argentina; and finally, we close this paper with some concluding remarks and future perspectives in section 5 .

\section{The LAGO Space Weather Program}

The LAGO project is organized around scientific and academic objectives [2] that are carried out within specific LAGO programs. The LAGO Space Weather (LAGO-SW) program is oriented toward studying how the variations of the flux of secondary particles at ground level can be linked with the heliospheric and geomagnetic modulation of GCRs, providing precise information of the changing conditions of the near-Earth space environment. These secondary particles are produced 


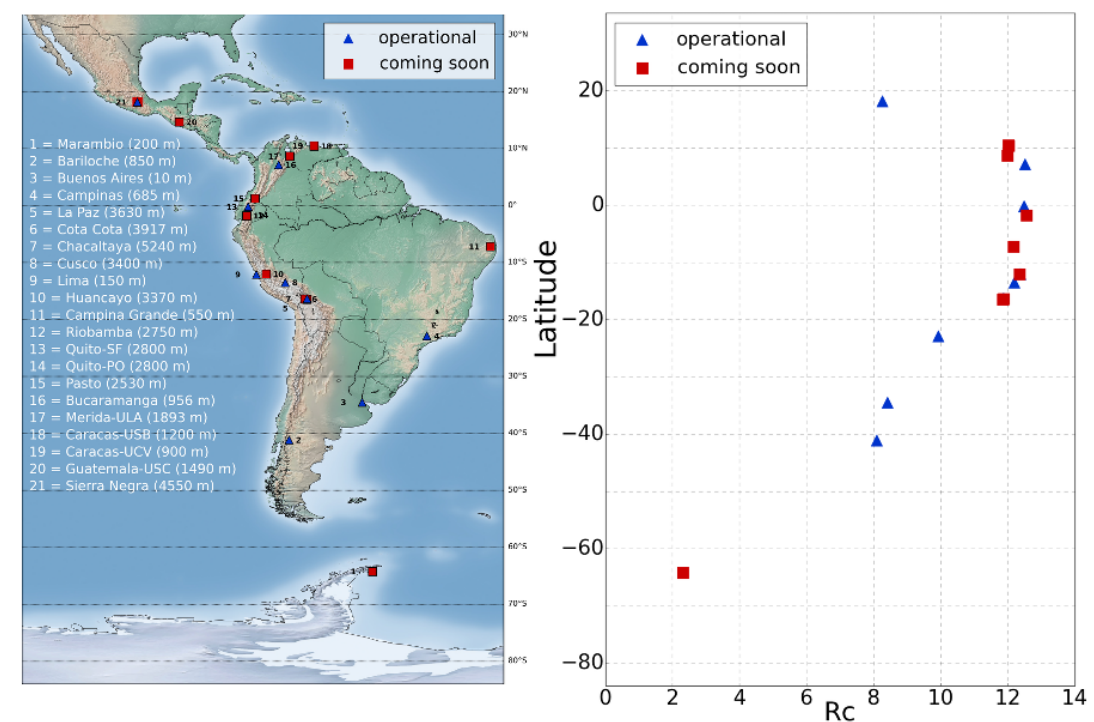

Figure 1: Geographical distribution and altitudes of the operational LAGO water Cherenkov detectors (blue triangles), and those that are being deployed and will start its operation during the 2015-2016 biennium (red squares). At the right panel, the vertical rigidity cut-off of each LAGO site are shown.

during the interaction of a GCR with the atmosphere and are measured at ground level by the LAGO detection network.

As will be shown in section 4 we are able to determine the flux of secondary particles at different bands of deposited energy in the detector by using pulse shape discrimination techniques. These bands are dominated by different components of the primary radiation. This is what we called the multi-spectral analysis technique (MSAT). Then, by combining all the data measured at different locations of our detection network, the LAGO project will provide very detailed and simultaneous information of the temporal evolution and of the small and large scales characteristics of the disturbances produced by different transient and long term space weather phenomena.

This complex problem comprises very different processes at different spatial and time scales. So, our approach is based on the following conceptual schema:

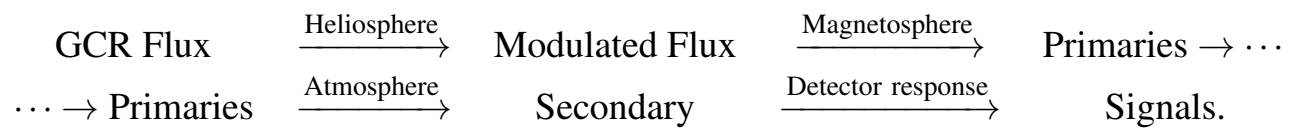

Each step of this chain is evaluated and token into account by direct measurements in our stations [3], or by accessing data from several online servers (e.g. the $\mathrm{D}_{\mathrm{st}}$ index, see section 3). All our measurements are complemented by a complete chain of simulations, as will be described in the next section.

\section{Cosmic rays, Extensive Air Showers and the Geomagnetic Field}

The LAGO Space Weather program is supported by an intensive and complete chain of simulations accounting for three important factors: the geomagnetic effects, the development of the extensive air showers (EAS) in the atmosphere, and the detector response to the different types of secondary particles at ground level by means of a GEANT4 model [6, 7]. 


\subsection{Galactic Cosmic Rays and the Geomagnetic Field}

The GF effects on the propagation of charged particles that could contribute to the background radiation at ground level can be characterized by the directional rigidity cut-off $R_{c}$ at each LAGO site, considered as a function of the geographical position at some altitude (Lat, Lon, Alt) and the arrival direction $(\theta, \phi)$ of the primary at this point. Additionally, a time stamp (TS YYMMDDHHMMSS UTC) should also be included to address transient phenomena during those periods of intense geomagnetic activity, so $R_{c} \equiv R_{c}$ (TS, Lat, Lon, Alt, $\left.\theta, \phi\right)$.

This directional $R_{c}$ can be calculated at each LAGO site by using the Magnetocosmics code [8] by applying the backtracking technique [9] and using the International Geomagnetic Field Reference (IGRF) version 11 [10] for modeling the near-earth GF $\left(r<5 R_{\oplus}\right)$ and the Tsyganenkov (TSY01) [11] to describe the outer GF. As it is commonly used, our description of the GF can be tagged by six parameters: solar wind dynamic pressure, $\mathrm{D}_{\mathrm{ST}}$ index, $B_{y}$ and $B_{z}$ components of the geomagnetic field (in GSM system), and the $G_{1}$ and $G_{2}$ parameters of TSY01 model.

As the next step in the simulation chain is performed in CORSIKA v 7.3500 [12], that accounts for GF effects during the particle propagation in the atmosphere, $R_{c}$ is calculated at the edge of the atmosphere, $\sim 110 \mathrm{~km}$ a.s.l. As an example, we will show the calculations performed for the LAGO site in Bucaramanga, Colombia $\left(+7^{\circ} 8^{\prime} \mathrm{LN},-73^{\circ} 0^{\prime} \mathrm{LW}\right)$ for May of 2005 (initial TS 0505090900). The changing conditions of the GF were obtained from data of the Virtual Radiation Belt Observatory ${ }^{1}$. Results of this calculations are displayed in figures 2: plate 2(a) shows the strong relation between the $R_{c}$ and the arrival direction of the charged particles hitting the edge of the Earth atmosphere; plate 2(b) displays the predicted changes on $R_{c}$ during the geomagnetic storm of May 15th 2005, particularly for azimuth angles greater than $150^{\circ}$. To look for the origin of the anomalous behavior observed in the $R_{c}$ for arrival directions in the range $45^{\circ}<\theta<90^{\circ}$ and $250^{\circ}<\phi<300^{\circ}$, we performed detailed analysis of the individual paths of the particles in this arrival direction range. We found that all these trajectories go through the South Atlantic Anomaly, showing that it could be responsible for this particular behaviour.

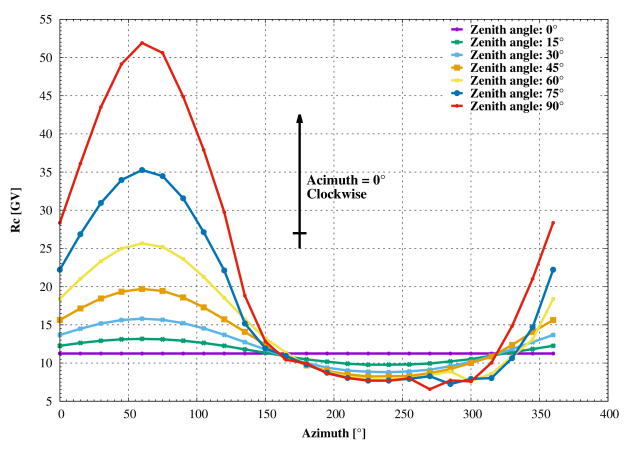

(a)

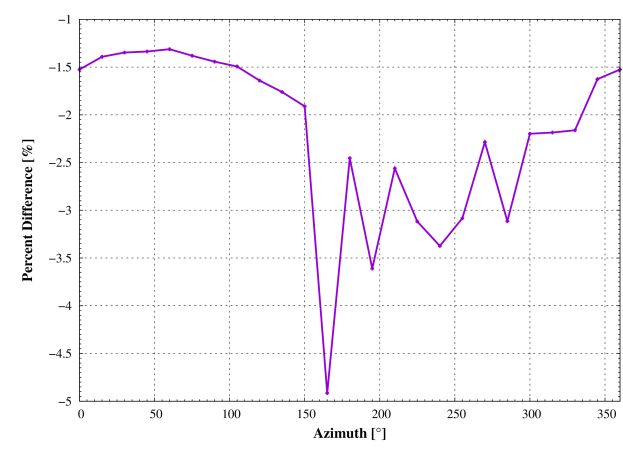

(b)

Figure 2: (a) Directional rigidity cut-off $\left(R_{c}\right)$ at the atmosphere edge, as a function of the azimuth angle $\phi$ and for different zenith angles $\theta$ for the LAGO site in Bucamaranga, Colombia, during secular conditions of the geomagnetic field. The effect of the SAA is observed for inclined particles in the range $250^{\circ}<\phi<300^{\circ}$. (b) Relative difference for $\theta=45^{\circ}$ between the $R_{c}$ under secular condition (left) and during the geomagnetic storm occurred on May 15th 2005 09:00 UTC.

\footnotetext{
${ }^{1}$ http://virbo.org
} 


\subsection{Secondary Particles Flux at Ground Level}

The second step of the chain is a complete simulation based in CORSIKA $v 7.3500^{2}$ [12] of the secondaries observed at ground level by the EAS produced during the interaction with the atmosphere of the complete flux of primaries. The flux of all nuclei primaries in the range $1 \leq Z \leq$ 26 was assumed to be uniform in solid angle and considering a single power law for each nuclei to describe the energy dependence in the range $Z \times R_{c} \leq E \leq 1 \mathrm{PeV}$ [13]. The corresponding parameters were obtained from [14]. As the $R_{c}$ depends on the arrival direction of each primary, the total number of primaries $N$ is strongly angular dependent. To avoid numerical issues during the calculation of $N$, we simulate all the primaries starting from the lowest $R_{c}$ and then we simply suppress from the final result those secondaries that were originated from primaries that should not be allowed to enter the atmosphere due the geomagnetic field effect.

The calculation performed without GF corrections can be considered as an approximation to the more precise flux of secondaries calculated when the effects of the geomagnetic perturbation have been considered. The effect of the geomagnetic field in the flux of primaries impinging the Earth atmosphere can be observed in figure 3(a), where a comparison of fluxes with/without GF before the edge of the atmosphere is shown. Only primaries that actually produced secondary particles at ground are considered. Low energy nuclei are affected, but it is not a sharp cutoff as the studied effect depends on its $Z$, on arrival directions of the primaries, and also includes the effect of atmospheric absorption of secondary particles during the EAS development.

The GF effect in the secondary flux at ground level can also be obtained by comparing the expected flux without corrections with the corrected corresponding one. In the figure 3(b), the relative difference between these two cases is shown as a function of the particle momentum. The observed differences are large enough to justify the incorporation of geomagnetic corrections when the low energy secondary flux is calculated. A large deviation in the total flux is also observed, which is peaked at $p_{\mathrm{sec}} \simeq 500 \mathrm{MeV} / \mathrm{c}$. At this values, the secondary flux is dominated by neutrons. For this particular component, the decrease at ground level due to GF effect in secular conditions represents a diminution of $-36.6 \%$. This could be an indication of the sensitivity of secondary neutron flux as a proxy of the changing conditions in the near-earth space environment.

Based on this preliminary results, different studies are conducted within the LAGO Collaboration to evaluate the sensitivity of WCD via neutron capture in pure water or to add dopants such as gadolinium-boron mixtures.

\section{Multi-spectral Analysis of Space Weather Phenomena}

In this section we shall show one of the actions of the LAGO Space Weather program which proves how a single WCD is able to detect transient solar phenomena affecting the flux of particles at the surface of the Earth. Previous works have shown WCD capabilities to study heliospheric phenomena [13], and here we exploit the possibilities to study the variations on the flux of secondaries at different bands of deposited energy $E_{d}$.

The charge histogram of the WCD, typically used for detector calibration, is obtained by time integration of the individual pulses measured in the WCD. The resulting histogram, showed in

\footnotetext{
${ }^{2}$ Options used: QGSJET-II-04; GHEISHA-2002; EGS4; Curve, External Atmosphere, Volumetric Detector
} 


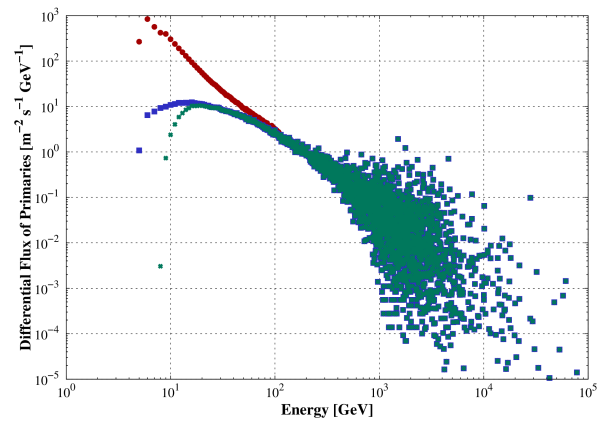

(a)

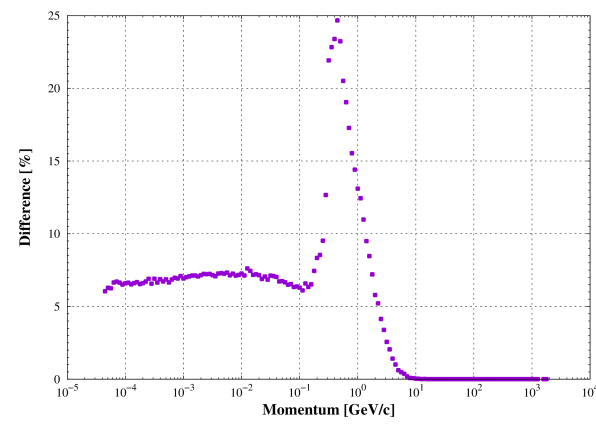

(b)

Figure 3: (a) Geomagnetic effects in the flux of low energy GCR, observed when comparing the flux of primaries that actually produced secondary particles at ground when GF corrections for secular conditions are included (green stars) and when they are not considered (blue squares). The total flux of injected primaries is also included (red circles). (b) The GF effect in the secondary particles at ground level as a function of the secondary momentum. In this curve, the relative difference between the flux without GF corrections and the corrected flux is shown. The larger differences are observed at $p_{\mathrm{sec}} \simeq 500 \mathrm{MeV} / \mathrm{c}$. In this region, the flux of secondaries is dominated by neutrons.

figure 4, is originated by the convolution of the response of the WCD to the different types of EAS particles and the water quality, the inner coating used, the PMT size and the detector geometry. By doing first principle calculations and detailed simulations, it is well established that the small signal region, corresponding to low values of $E_{d}$, is dominated by the electromagnetic component (EM, $\gamma_{\mathrm{S}}$ and $e^{ \pm}$) of the shower; while high values of $E_{d}$ correspond to the simultaneous entrance (within the electronic sampling time, $10 \mathrm{~ns}-40 \mathrm{~ns}$ ) of multiple particles to the detector volume (the so called mini-shower - MS - regime). Finally, intermediate values of $E_{d}$, evidenced by a characteristic peak ${ }^{3}$ in the histogram called the muon hump, are dominated by single muons through the detector.

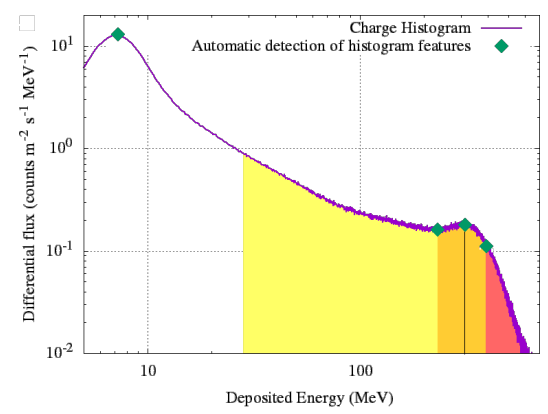

Figure 4: Charge histogram of one LAGO WCD in Bariloche, Argentina. A fully automated algorithm look for histogram features (green diamonds) to define integrations bands (shaded regions). Each integration band is dominated by different type of particles: EM particles (yellow), muons (orange) and multiple particles (pink).

The LAGO MSAT determines the evolution of the flux observed at those three bands: EMband, $\mu$-band and MS-band. As the transition points between these different regimes are characterized by changes in the histogram slopes, a fully automated algorithm searches for all these features in the 1-hour calibration histograms and determines the integrated total flux in those three bands as can be seen in figure 4 . Typical integration windows are between 1 minute and 15 minutes long.

\footnotetext{
${ }^{3}$ The first peak seen at the histogram at very low signals is originated by the detector trigger system and is not considered in these analysis.
} 
As a first attempt, we applied the MSAT to analyze the Forbush event observed on March 8th, 2012, associated with the passage of an iCME and detected by different instruments [15]. The studied data were acquired in a single $1.8 \mathrm{~m}^{2} \mathrm{WCD}$ (with $2.5 \mathrm{~m}^{3}$ of pure water and a 8 " Hamamatsu R5912 PMT) at the LAGO site of Bariloche, Argentina. After applied flux pressure correction to each band, the FD is clearly visible in our MSAT, with a maximum peak-to-peak decrease of $\sim 5 \%$, $\sim 6 \%$, and $\sim 4 \%$ in the EM, $\mu$ and MS bands respectively. The temporal evolution of the EM and $\mu$ bands are shown in figure 5, where our data is compared with data from the Rome neutron monitor ${ }^{4}$. Solar daily modulation of the flux is also visible on both bands. The particular features observed in our data are currently under careful analysis and will be published soon.

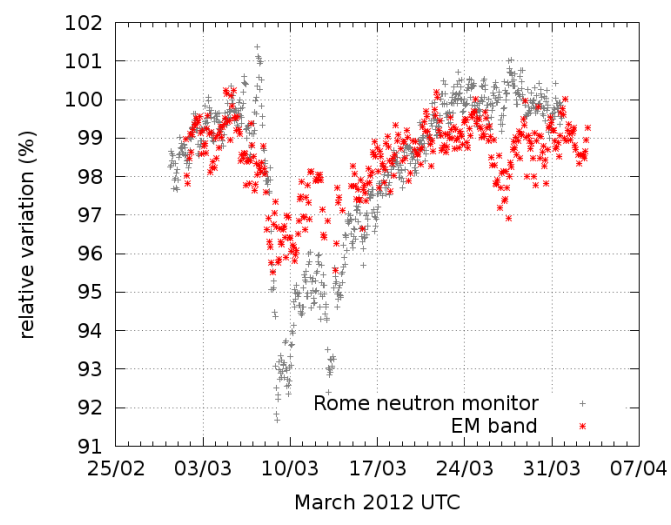

(a)

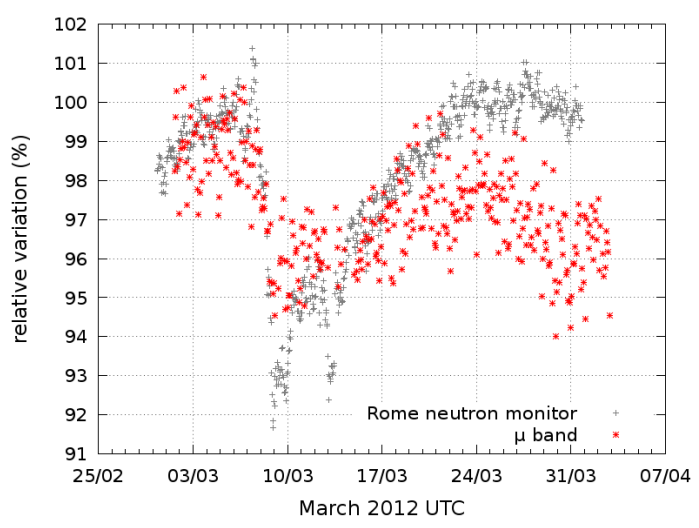

(b)

Figure 5: Results of Multi-Spectal Analysis of the Forbush Decrease of March 8th, 2012 measured in a single $1.8 \mathrm{~m}^{2}$ WCD installed in Bariloche, Argentina (red stars), compared with measurements of the Rome neutron monitor (gray pluses), in two bands: EM-band (a), and $\mu$-band (b).

\section{Summary and Conclusions}

In this seminal paper we describe the basis of the LAGO Space Weather program, that accounts for a complete chain of measurement and simulations to study Space Weather phenomena from ground level.

Two important contributions have been presented. First, the simulation steps introduced to obtain a precise calculation of the secondary particle flux at ground level for any current or future LAGO site, that includes now a method to account for geomagnetic effects in the flux of secondaries. We find significant differences on the expected flux of particles at ground level when GF corrections are considered. At this moment, simulations of the background flux expected under different geomagnetic conditions for all LAGO sites are underway.

The second contribution proves the capabilities of single WCD to detect transient space weather phenomena from ground level. The introduction of the MSAT shows also that using even single detector it is possible to determine the flux at bands that are dominated by different types of particles coming from different primaries contribution.

The principal result of this work is, however, to stablish the conceptual basis of one of the main objectives of the LAGO project: the observation of space weather phenomena from ground

\footnotetext{
${ }^{4}$ Available at the NEST repository, http://www.nmdb.eu/nest
} 
level. The LAGO detection network, once it is completed and operational, will provide the unique information to delve in the very complex problem of the Sun-Earth connection.

Acknowledgments: The LAGO Collaboration is very thankful to the Pierre Auger Collaboration for its continuous support. LAN and YP gratefully acknowledges the partial financial support from CDCHT-ULA project C-1598-08-05-A. CSC, LAN, MSD and HA are also in debt to Vicerrectoría Investigación y Extensión Universidad Industrial de Santander for its permanent support.

\section{References}

[1] H. Cane, Coronal Mass Ejections and Forbush Decreases, Space Science Reviews 93 (2000), no. 1-2 $55-77$.

[2] H. Asorey for the LAGO Collaboration, LAGO: the Latin American Giant Observatory, this proceedings (2015).

[3] I. Sidelnik and for the LAGO Collaboration, The sites of the Latin American Giant Observatory, this proceedings (2015).

[4] A. Galindo and others for the LAGO Collaboration, Sensitivity of LAGO and Calibration of a Water-Cherenkov Detector in Sierra Negra, México., this proceedings (2015).

[5] S. Dasso and others for the LAGO Collaboration, A project to install water-cherenkov detectors in the antarctic peninsula as part of the LAGO detection network, this proceedings (2015).

[6] L. Otiniano and F. Quispe for the LAGO Collaboration, Development of a High Altitude LAGO Site in Peru, this proceedings (2015).

[7] S. Vargas and others for the LAGO Collaboration, LAGO Ecuador, Implementing a Set of WCD Detectors for Space Weather Research: first results and further developments, this proceedings (2015).

[8] L. Desorgher, Magnetocosmics: Geant4 application for simulating the propagation of cosmic rays through the Earth magnetosphere, 2003.

[9] J. J. Masías-Meza and S. Dasso, Geomagnetic effects on Cosmic Ray propagation under different conditions for Buenos Aires and Marambio, Argentina, Sun and Geosphere 9 (2014) 41-47.

[10] I. A. of Geomagnetism and W. G. V.-M. Aeronomy, International Geomagnetic Reference Field: the eleventh generation, Geophysical Journal International 183 (2010), no. 3 1216-1230.

[11] N. A. Tsyganenko, A model of the near magnetosphere with a dawn-dusk asymmetry 1. Mathematical structure, Journal of Geophysical Research: Space Physics 107 (2002), no. A8 SMP 12-1-SMP $12-15$.

[12] D. Heck, J. Knapp, J. N. Capdevielle, G. Schatz, and T. Thouw, CORSIKA: a Monte Carlo code to simulate extensive air showers. Feb., 1998.

[13] H. A. for the LAGO Collaboration, The LAGO Solar Project, Proc. 33th ICRC, Rio de Janerio, Brazil (2013).

[14] P. K. F. Grieder, Cosmic Rays at Earth. Researcher's Reference Manual and Data Book. Elsevier Science, Institute of Physics University of Bern Bern Switzerland, 2001.

[15] V. Alekseenko, F. Arneodo, G. Bruno, W. Fulgione, D. Gromushkin, O. Shchegolev, Y. Stenkin, V. Stepanov, and V. Sulakov, Registration of Forbush decrease 2012/03/08 with a global net of the thermal neutron scintillation, Journal of Physics: Conference Series 409 (2013), no. 1012190. 